Printed in Great Britain

\title{
Mitotic Cycle of the Kala-Azar Parasite, Leishmania donovani
}

\author{
By JYOTSNA CHAKRABORTY AND N. N. DAS GUPTA \\ Biophysics Division, Saha Institute of Nuclear Physics, Calcutta-9, India
}

(Received 25 September 1961)

\begin{abstract}
SUMMARY
The growth rate and the time schedule of the mitotic cycle of the leptomonads of Leishmania donovani, the Kala-azar parasite, were studied. The numbers of cells in the different division stages were estimated by photomicroscopy after Feulgen staining. The observed time intervals of resting, prophase, metaphase, anaphase, telophase and binucleate condition were $15.2,1 \cdot 1,3.9,1 \cdot 1,0.9$ and $1.8 \mathrm{hr}$., respectively; the total generation time was $24 \mathrm{hr}$.
\end{abstract}

\section{INTRODUCTION}

The fine structure organization of the protozoon Leishmania donovani, the Kalaazar parasite, has been the subject of several recent investigations (Chang, 1956; Inoki, Nakanishi, Nakabayashi \& Ohno, 1957; Inoki, Nakanishi \& Nakabayashi, 1958; Pyne, 1958; Pyne \& Chakraborty, 1958; Chakraborty \& Das Gupta, 1960), but no study has so far been made on the time schedule of the mitotic cycle of this parasite. A precise measurement of the time schedule of the different stages of the mitotic cycle has now been made from photomicroscopic and electron microscopic investigations, combined with a measurement of the mean generation time.

\section{METHODS}

The leptomonad form of Leishmania donovani was cultured in NNN medium (Nicolle 1908) in this laboratory. NNN medium is a solid medium with a little water of condensation on the top of the slant. The organisms crowd together in this water of condensation.

Determination of the mean generation time. The water of condensation from a stock culture tube of NNN medium containing the organisms was mixed with about $2 \mathrm{ml}$. normal saline. This mixture was equally distributed into each of $\mathbf{3 0}$ culture tubes, which were then incubated at $\mathbf{2 2}^{\circ} \pm 1^{\circ}$. At the end of $24 \mathrm{hr}$., the water of condensation containing the organism was carefully pipetted into a centrifuge tube. Some organisms remained attached to the agar surface which were washed with a little normal saline and collected in the centrifuge tube. The saline solution containing the organisms was centrifuged at about $\mathbf{3 0 0 0} \mathrm{rev} . / \mathrm{min}$. The organisms settled down, and the supernatant fluid was discarded. The sediment containing only the organisms was diluted with a measured volume of normal saline, then a loop of organism was put on the coverslip on which a drop of procaine solution was previously dried for immobilization of the organisms. The coverslip was then inverted and placed over the haemocytometer slide and the total number determined by the usual procedure. Estimation of cell viability was made by direct observations with optical microscopy. 
In exponentially growing cultures young organisms are more numerous than old ones owing to the division of one organism into two daughters. On account of this fact the fraction of the average life span of a cell, spent in any stage, is not simply equal to the proportion of cells observed in that phase; a correction has to be made. It has been shown by Powell (1956) and Edwards et al. (1960) that if c.P.I. is the cumulative phase index, i.e. fraction of all cells between division and a particular phase of growth, and $X$ the cumulative fraction of time between division up to and including the particular phase in question then

or

$$
\text { C.P.I. }=2\left(1-2^{-x}\right) \text {, }
$$

$$
x=1-\frac{\log (2-\text { C.P.I. })}{\log _{2}} \text {. }
$$

With the help of this equation the durations of the different phases of the mitotic cycle were estimated. The variation in the number of counts with days was recorded. From a logarithmic plot of the number of the organisms against time after inoculation, the mean doubling time could be deduced in the usual manner as shown in Fig. 1.

Identification of the phases of the mitotic cycle. Specimens were examined by the optical microscope and by the electron microscope. For optical microscope studies leptomonads of Leishmania donovani from 4- to 7-day cultures were fixed for $2 \mathrm{hr}$. in the following fixative: chromic acid, 1 g.; $\mathrm{HgCl}_{2}, 2 \mathrm{~g}$; glacial acetic acid, $2 \mathrm{ml}$; water to $100 \mathrm{ml}$. After fixation, the specimens were hydrolysed in $\mathrm{N}-\mathrm{HCl}$ at $60^{\circ}$ for $7 \mathrm{~min}$. and stained with Feulgen solution for $45 \mathrm{~min}$. Counterstaining was done in $\mathbf{0 . 2} \%(\mathrm{w} / \mathrm{v})$ Light Green (Gurr's; George T. Gurr Ltd., London, S.W. 6) dissolved in absolute ethanol for $15 \mathrm{~min}$. The specimens were then photomicrographed at a magnification of about $\times 1000$. For electron microscope study the organisms were first washed thoroughly in normal saline to remove impurities and then fixed in $1 \%(\mathrm{w} / \mathrm{v})$ osmium tetroxide, $\mathrm{pH} \mathrm{7 \cdot 4}$, for $30 \mathrm{~min}$. (Palade, 1952; Sjostrand, 1956). After fixation the organisms were dehydrated through the 30, 50, 70, 90 and $100 \%$ ethanol and then embedded in a mixture of 4 parts of butyl methacrylate monomer, 1 part of methyl methacrylate monomer $(\mathrm{v} / \mathrm{v})$ and $2 \%$ $(w / v)$ Luperco CDB. After polymerization (24 hr. at $\left.48^{\circ}\right)$, sections were cut with a Porter Blum microtome and the specimens were examined with a Siemens Elmiskop I at $60 \mathrm{kV}$.

\section{RESULTS}

\section{Characteristics of the growth curve}

Changes in the total number of the organisms at different times following inoculation are shown in Fig. 1. Theorganism multiplied exponentially from the second day onwards. The observed doubling time during the exponential growth phase, as shown in Fig. 1, was estimated to be $24 \mathrm{hr}$. This is equivalent to the average life span of the cells in the culture. The mean generation time found here agrees with that observed by Chang \& Negherbon (1947) who used a different medium. All Leishmania were found to remain viable throughout the 10 days of the growth curve. The counting, however, could not be continued beyond the tenth day since most of the organisms then formed a rosette and it was very difficult to count them. Also it was observed that after this period some organisms were dead or dying. 


\section{Duration of the phases of the mitotic cycle}

Observations with the optical microscope. About 400 specimens in which the nuclear division stage could be clearly established were observed with the optical microscope after Feulgen staining. Most of these specimens were taken from cultures from the middle of the log phase, i.e. 6 days after inoculation. A few typical photomicrographs obtained after the Feulgen staining are shown in Plates 1 and 2,

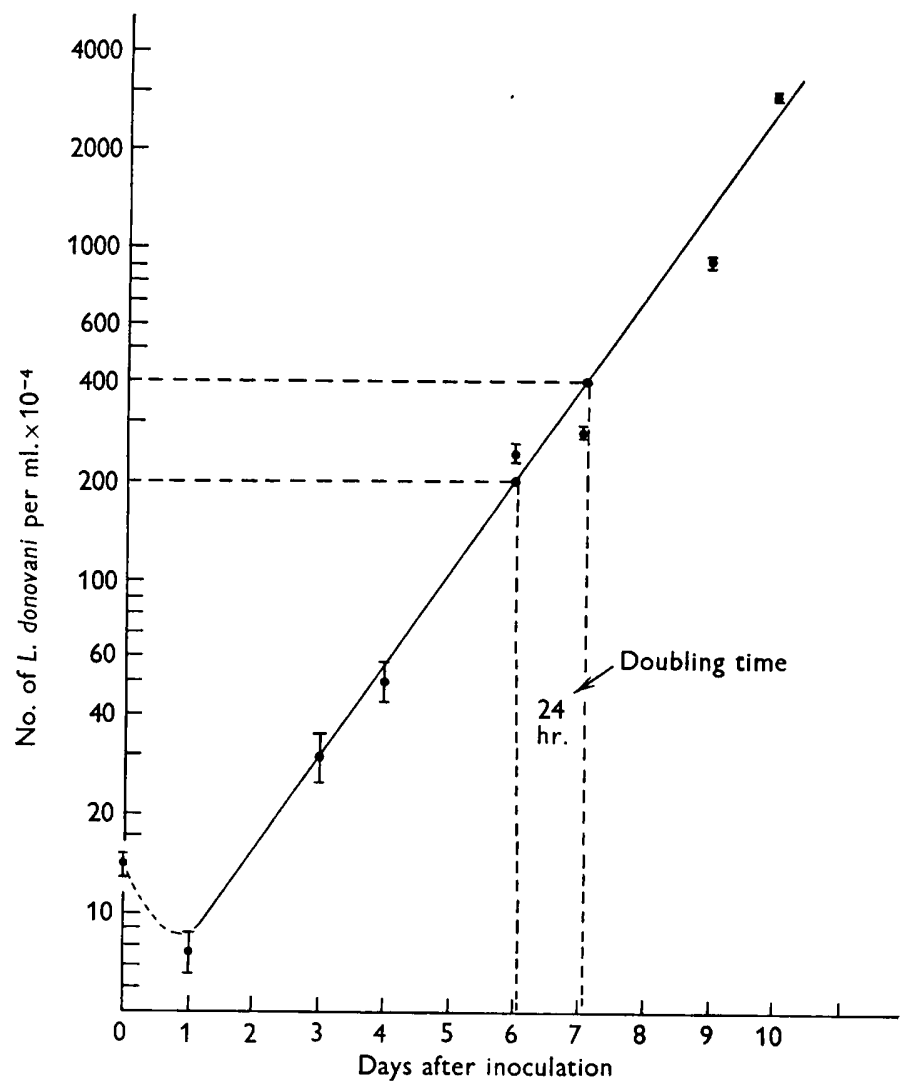

Fig. 1. Growth curve of Leishmania donovani in NNN medium at $22^{\circ} \pm 1^{\circ}$.

Table 1. Duration of the phases in the mitotic cycle of the leptomonad form of Leishmania donovani during log phase of growth

$\begin{array}{lcccc}\begin{array}{c}\text { Nuclear } \\ \text { phase }\end{array} & \begin{array}{c}\text { Phase } \\ \text { incidence } \\ \text { (\%) }\end{array} & \begin{array}{c}\text { Cumulative } \\ \text { phase } \\ \text { index } \\ \text { (c.P.I., \%) }\end{array} & \begin{array}{c}\text { Duration } \\ \text { of each } \\ \text { phase } \\ \text { (hr.) }\end{array} & \begin{array}{c}\text { Time from } \\ \text { beginning } \\ \text { of cycle } \\ \text { (hr.) }\end{array} \\ \text { Interphase } & \mathbf{7 1} & \mathbf{7 1} & \mathbf{1 5 . 2} & \mathbf{1 5 . 2} \\ \text { Prophase } & \mathbf{4} & \mathbf{7 5} & \mathbf{1 . 1} & \mathbf{1 6 . 3} \\ \text { Metaphase } & \mathbf{1 3 . 5} & \mathbf{8 8 . 5} & \mathbf{3 . 9} & \mathbf{2 0 . 2} \\ \text { Anaphase } & \mathbf{3 . 5} & \mathbf{9 2} & \mathbf{1 . 1} & \mathbf{2 1 \cdot 3} \\ \text { Telophase } & \mathbf{2 . 5} & \mathbf{9 4 . 5} & \mathbf{0 . 9} & \mathbf{2 2 . 2} \\ \text { Binucleate } & \mathbf{5 . 5} & 100 & \mathbf{1 . 8} & \mathbf{2 4 . 0}\end{array}$


figs. $1 \mathrm{~A}$ to $6 \mathrm{~A}$. Mean values for the numbers of organisms and the duration of the different phases of the division cycle are shown in Table 1.

Observation with the electron microscope. The nuclear division stages observed with the optical microscope after Feulgen staining were strikingly confirmed by the electron micrographs of the ultra-thin sections. The size of the nucleus of this parasite is small and the individual chromosomes at metaphase are almost at the limit of resolution of the optical microscope. The electron micrographs show the details of the division stages more clearly.

Plate 1 , fig. 1 , is the resting nucleus within which a single prominent nucleolus is visible. Plate 1, fig. 2, represents a prophase nucleus in which the nucleolus has disappeared and the chromosomes have just become visible. Plate 1 , fig. 3 , is the polar view of the metaphase stage, with the 8 chromosomes arranged in the equatorial plane. Plate 2, fig. 4, is the anaphase stage showing the separated chromosomes moving towards the opposite poles. Plate 2, fig. 5, shows the telophase nucleus with a slight constriction in the nuclear wall at the equator and Plate 2, fig. 6, represents the binucleate condition where the nuclear division is completed with the formation of two daughter nuclei, but the organism is not yet divided. Such electron micrographs confirm the division stages observed with the optical microscope and represented in PIs. 1 and 2, figs. $1 \mathrm{~A}$ to $6 \mathrm{~A}$. About 70 good electron micrographs were obtained which show the organism in different stages of nuclear division. The number of organisms in the different stages were counted from these electron micrographs. Within the limitations of the sectioning technique, the frequency of occurrence of the different stages agreed with those derived from optical microscopy.

The authors are indebted to Ministry of Scientific Research and Cultural Affairs, Government of India, for the financial assistance. Thanks are also due to Mr M. L. De for help in taking the electron micrographs and many valuable discussions.

\section{REFERENCES}

Chakraborty, J. \& Das Gupta, N. N. (1960). Ultrastructure of the pellicle and the nucleus of Leishmania donovani. 4th int. Conf. Elec. Micros. 2, 510.

Chang, P. C. H. (1956). The ultrastructure of Leishmania donovani. J. Parasit. 42, 126.

Chang, S. L. \& Negherbon, W.O. (1947). Studies on Haemoflagellates. II. A study of the growth rates of Leishmania donovani, L. brasiliensis, L. tropica and Trypanosoma cruzi in culture. J. infect. Dis. 80, 172.

Edwards, J. L., Koch, A. L., Yoncis, P., Freese, H. L., Laite, M. B. \& Donalson, J. T. (1960). Some characteristics of DNA synthesis and the mitotic cycle in Ehrlich ascites tumor cells. J. biophys. biochem. Cytol. 7, 273.

Inoki, S., Nakanishi, K., Nakabayashi, T. \& Ohno, M. (1957). Fine structure of the flagellum of Leishmania donovani revealed by electron microscope. Med.J. Osaka Univ. 7,719 .

Inoki, S., Nakanishi, K. \& Nakabayashi, T. (1958). Study of Leishmania donovani with special reference to the kinetoplast, mitochondria and golgi zone by electron microscope employing the thin section technique. Biken's J., Japan, 1, 194.

Nicolle, C. (1908). Nouvelles acquisitions sur le Kala-azar: cultures, inoculations au chien, étiologie. C.R. Acad. Sci. Paris, 146, 498.

Palade, G. E. (1952). A study of fixation for electron microscopy. J. exp. Med. 95, 285.

Powell, E. O. (1956). Growth rate and generation time of bacteria with special reference to continuous culture. J. gen. Microbiol. 15, 492. 

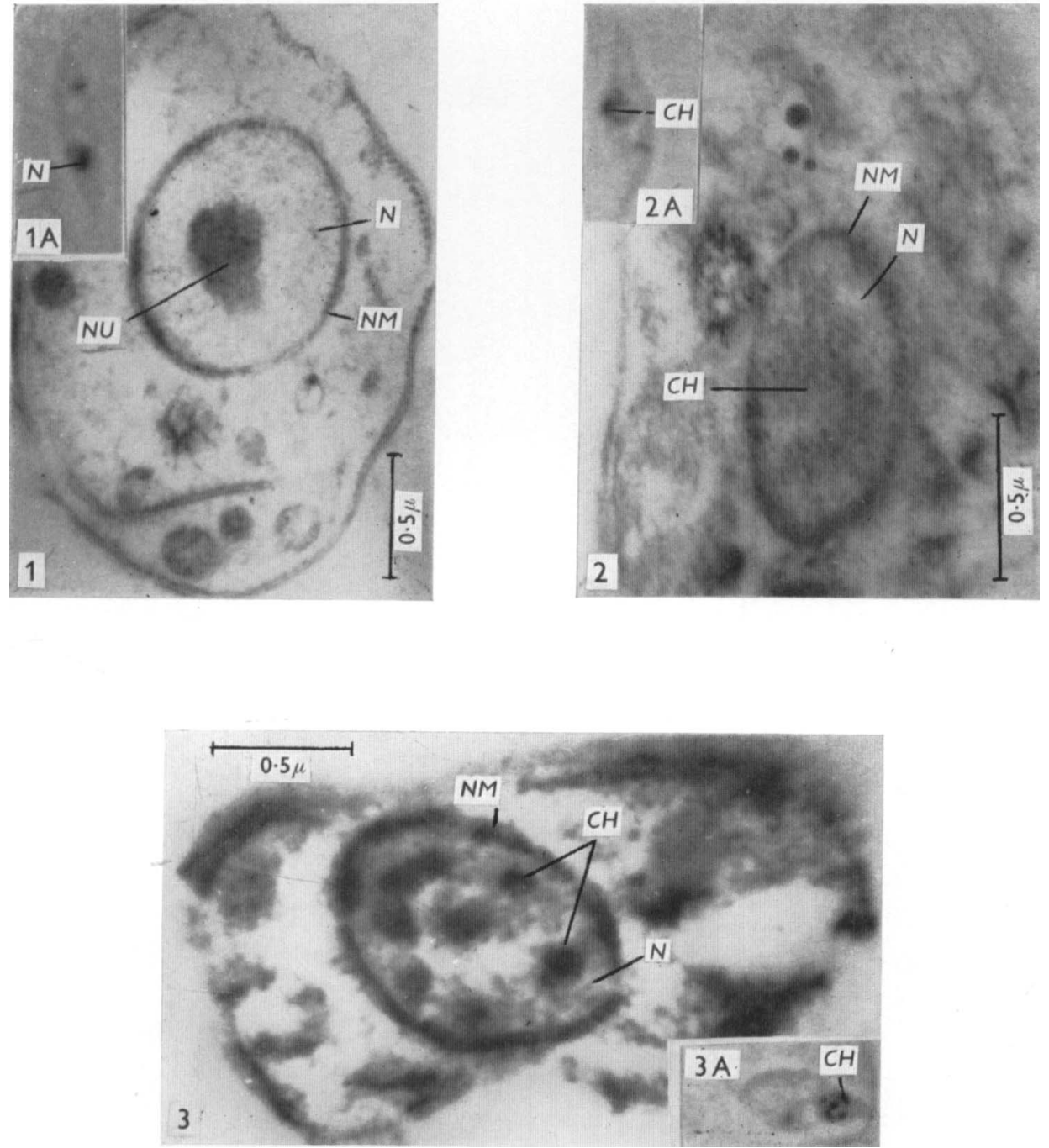

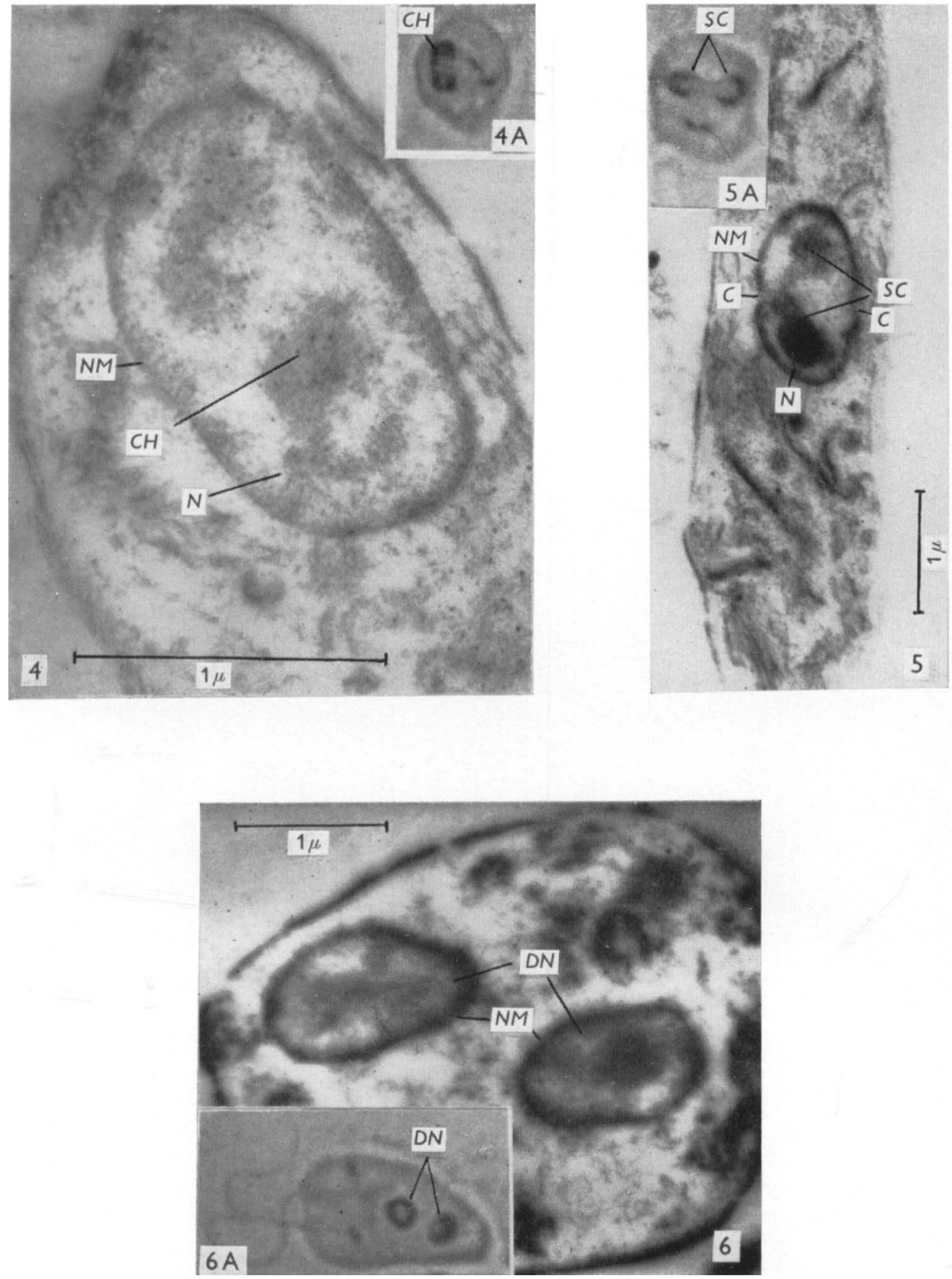
Pyne, C. K. (1958). Electron microscopic investigations on the leptomonad form of Leishmania donovani. Exp. Cell Res. 14, 388.

Pyne, C. K. \& Chakraborty, J. (1958). Electron microscopic studies on the basal apparatus of the flagellum in the protozoon, Leishmania donovani. J. Protozool. 5, 264. SJostrand, F. S. (1956). Electron microscopy of cells and tissue. In Physical Technique in Biological Research, Vol. III, chap. 6, 241. New York: Academic Press Inc.

\section{EXPLANATION OF PLATES}

Plates 1 and 2. Figs. 1-6, electron micrographs of the different division stages; magnifications indicated by a $\mu$ mark. Figs. $1 \mathrm{~A}-6 \mathrm{~A}$, photomicrographs (light microscope) of the corresponding stages after Feulgen staining. $\times \mathbf{3 0 0 0}$.

\section{Plate 1}

Figs. 1 and 1 A. Resting stage of the nucleus $(N)$ of $L$. donovani, showing the prominent nuclear membrane $(N M)$ and a single irregularly shaped nucleolus $(N U)$.

Figs. 2 and $2 \mathrm{~A}$. The nucleus in prophase stage. The chromosomes $(\mathrm{CH})$ are just visible but the nucleolus is absent.

Figs. 3 and $3 \mathrm{~A}$. The nucleus in the metaphase stage. The chromosomes $(\mathrm{CH})$ are arranged at the equatorial plate (polar view).

\section{Plate 2}

Figs. 4 and 4A. Anaphase nucleus with separated daughter chromosomes $(\mathrm{CH})$ moved away from the equator.

Figs. 5 and 5A. Telophase nucleus with separated chromosomes $(S C)$ at the opposite poles. A slight constriction $(C)$ at the equatorial region of the nuclear membrane $(N M)$ is also visible.

Figs. 6 and $6 \mathrm{~A}$. Binucleate condition of the organisms, with two daughter nuclei $(D N)$ in each body. 